\title{
Adipose tissue derived mesenchymal stem cell transplantation in the treatment of ischemia/reperfusion induced acute kidney injury in rats. Application route and therapeutic window ${ }^{1}$
}

Betânia Souza Monteiro', Bianka Souza dos Santos", Bruna Lopes de Almeida", Emy Hiura", Wagner Alexey Back Fiorio"', Gisele Pereira Valdetaro", Dariele Vieira Gonçalves", Caroline Saraiva Silva'v, Tatiana Championv ${ }^{\mathrm{V}}$ Daniela Campagnolv

'Full Professor, Stem Cell and Cellular Therapy Laboratory, Animal Science Program, Universidade Vila Velha (UVV), Brazil. Conception and design of the study, manuscript writing, critical revision, final approval.

"Veterinary Medical, Stem Cell and Cellular Therapy Laboratory, Animal Science Program, UVV, Vila Velha-ES, Brazil. Technical procedures, acquisition of data, manuscript preparation and writing.

"'Veterinay Medical, Advanced Imaging Center and Veterinary Pathology, Vila Velha-ES, Brazil. Histopathological examinations.

IVeterinay Medical, PetScan Diagnoses Center, Vila Velha-ES, Brazil. Imagens examinations.

${ }^{\vee}$ Assistant Professor, Veterinary Department, Universidade Federal da Fronteira Sul (UFFS), Realeza-PR, Brazil. Statistics analysis, critical revision, final approval.

"Veterinay Medical, Animal Experimentation Division, Clinical Hospital, Universidade Federal do Rio Grande do Sul (UFRS), Porto Alegre-RS, Brazil. Substantive scientific and intellectual contributions to the study, critical revision.

\begin{abstract}
Purpose: To evaluate renal repair in rats who had renal infarction induced by the obstruction of blood flow in the renal artery and were treated with transplantation of adipose tissue derived mesenchymal stem cell

Methods: 16 -week-old Wistar rats $(n=72)$ were used, submitted to celiotomy and had of the renal artery and vein clipped for 24 hours. The animals were randomly assigned to 10 experimental homogeneous groups, corresponding to the treatments with phosphatebuffered saline (PBS) or adipose tissue derived mesenchymal stem cell (ADSC), duration of application (24 or 48 hours), and site of transplantation (lateral vein of the tail or intrarenal). After the treatments were performed, at 8 and 31 days, four animals in each group were subjected to left nephrectomy for histological studies.

Results: Histologically, a higher amount of cell debris and tubules devoid of the epithelium and a higher degree of necrosis were observed in the groups treated with PBS, as opposed to a low degree of necrosis and higher tubular vascularization in the groups treated with ADSC, particularly in the group treated with intrarenal ADSC 48 hours after injury.

Conclusion: The transplantation of ADSC positively contributed to the replacement of necrotic tissue by renal tubular cells, vascularization of the renal parenchyma, and restoration of the organ function.
\end{abstract}

Key words: Cell and Tissue-Based Therapy. Ischemia. Reperfusion. Acute Kidney Injury. Rats. 


\section{Introduction}

Renal disease is a major cause of morbidity and mortality in human and animal patients. This disease requires expensive treatments, including drugs, hospitalizations, hemodialysis, and/or transplantation, whose results are not always satisfactory ${ }^{1}$. One cause of this disease is ischemia and subsequent necrosis, apoptosis of the tubular cells, and change in the polarity and integrity of the tubular barrier. The kidney initiates a process of repair that is dependent on the extension of the injury and on the time elapsed after ischemic injury ${ }^{2}$.

Research on cell therapy with the aim of finding solutions for the limited renal repair potential and providing a viable alternative for the treatment of renal injuries is ongoing worldwide. Some studies have suggested that transplantation of mesenchymal stem cells (MSC) contributes to renal repair, mainly via paracrine and telecrine mechanisms ${ }^{3,4}$. The research involving repair therapies using stem cells is in constant progress; the obtained results and the therapeutic potential of these cells seem promising ${ }^{5}$.

These promising results and the questions that still need to bead dressed, namely regarding the repairing and immunosuppressive ability of these cells, justify conducting new studies to investigate and clarify the role of MSC in restoring renal function. The present study aimed to assess renal repair in rats who had renal infarction induced by the obstruction of blood flow in the renal artery and were treated with transplantation of adipose tissue derived mesenchymal stem cell (ADSC), 24 or 48 hours after ischemia, via the intrarenal or intravenous route.

\section{Methods}

The experiments were approved by the
Animal Research and Experimentation Ethics Committee, protocols 109/2010.

In the in vitro experiment, three Lewis rats transgenic for green fluorescent protein (GFP) with 4-week-old male animals were used. In the in vivo experiment, 16-week-old female Wistar rats (Rattus norvegicus) $(n=72)$, weighing between 200 and 300g, were used.

\section{Cell culture and osteogenic differentiation}

The ADSC used in this project were processed of accords with protocols preestablishment for laboratory routine, preconized for Monteiro et al. ${ }^{6}$.

\section{Cell characterization}

An aliquot of adipose tissue-derived cells of the fourth passage was characterized by flow cytometry by analyzing the expression of cell surface molecules for MHC-I, MHC-II, CD 73 (anti-CD73 clone 5 F/B9 mouse; AbCam Cambridge, Massachusetts, USA), CD 11 (antiCD11b; AbCam), CD 90 (anti-CD90 clone Ox-7 mouse; AbCam), and CD 45 (anti-CD45 clone 69 mouse; BD Bioscience, San Jose, California, USA) using a FAC Scan flow cytometer and the CellQuest ${ }^{\circ}$ software.

\section{Preparation of cells for transplantation}

After the fourth passage, cells were trypsinized, observed by optical microscopy to assess cell viability, counted in a Newbauer chamber, and aliquoted at a concentration of $1.2 \times 10^{6}$ cells $/ 0.5 \mathrm{~mL}$ of PBS. Aliquots were transferred to $1.0 \mathrm{~mL}$ syringes. These were stored in a thermal box at room temperature and transported to the surgical center to be used in transplantation.

Anesthetic and surgical procedures and treatments

As pre anesthetic medication, the 
animals received a combination of ketamine $5 \%(20.0 \mathrm{mg} / \mathrm{kg})$, midazolam $0.5 \%(2.5 \mathrm{mg} / \mathrm{kg})$, and morphine $1 \%(1.0 \mathrm{mg} / \mathrm{kg})$, administered via the intramuscular (IM) route. After loss of righting reflex, trichotomy was performed in the abdominal region and was followed by anesthetic induction and maintenance with isoflurane $2.0 \%$ diluted in $100 \%$ oxygen via a facial mask coupled to a circuit with inhalation. The anesthetic plan was adjusted according to the absence of movements and the loss of tail and toe pinch reflex. Prophylactic antibiotic therapy was initiated with enrofloxacin (10.0 $\mathrm{mg} / \mathrm{kg}$ ) via the subcutaneous (SC) route.

The animals were subjected to paramedian preumbilical celiotomy, $2.0 \mathrm{~cm}$ in length. Following this, the autostatic retractor (blepharostat) was used to keep the cavity exposed, the left kidney was isolated, and the renal vessels were identified. The renal artery and vein were obstructed for 24 hours using a titanium atraumatic hemostatic clip (aneurysm clip).

Subsequently, the oblique muscles and skin were sutured using a synthetic non absorbable thread (nylon 3-0) and cross stitches $(X)$ and Wolf type stitches, respectively. In addition, surgical glue (cyanoacrylate) was applied on the skin suture. The animals that exhibited blood loss during the surgery intraperitoneally received up to $3.0 \mathrm{~mL}$ of saline solution (sodium chloride $0.9 \%$ ) before suturing.

After a period of 24 hours, the animals were again subjected to paramedian preumbilical celiotomy, and the hemostatic clip was removed. The surgical planes were again sutured. In the postoperative period, the animals received morphine sulfate, at a dose of $0.6 \mathrm{mg} / \mathrm{kg}$ via the IM route, every 8 hours, for 3 days. The animals were assigned to 10 distinct groups, according to the description below:

Group 1: Phosphate buffered saline
(PBS): eight animals treated with $0.5 \mathrm{~mL}$ of PBS administered in the lateral vein of the tail at 24 hours after reperfusion;

Group 2: PBS: eight animals treated with $0.5 \mathrm{~mL}$ of PBS administered via the intrarenal route at 24 hours after reperfusion;

Group 3: ADSC: eight animals treated with cell pellet containing $1.2 \times 10^{6}$ cells $/ 0.5 \mathrm{~mL}$ of PBS administered in the lateral vein of the tail at 24 hours after reperfusion;

Group 4: ADSC: eight animals treated with cell pellet containing $1.2 \times 10^{6}$ cells $/ 0.5 \mathrm{~mL}$ of PBS administered via the intrarenal route at 24 hours after reperfusion;

Group 5: PBS: eight animals treated with $0.5 \mathrm{~mL}$ of PBS administered in the lateral vein of the tail at 48 hours after reperfusion;

Group 6: PBS: eight animals treated with $0.5 \mathrm{~mL}$ of PBS administered via the intrarenal route at 48 hours after reperfusion;

Group 7: ADSC: eight animals treated with cell pellet containing $1.2 \times 10^{6}$ cells $/ 0.5 \mathrm{~mL}$ of PBS administered in the lateral vein of the tail at 48 hours after reperfusion;

Group 8: ADSC: eight animals treated with cell pellet containing $1.2 \times 10^{6}$ cells $/ 0.5 \mathrm{~mL}$ of PBS administered via the intrarenal route at 48 hours after reperfusion;

Group 9: Sham: four animals subjected only to the anesthetic procedures, followed by paramedian preumbilical celiotomy, renal manipulation, and suturing of the surgical planes, without artery clipping and blood flow obstruction;

Group 10: Histological control: four animals subjected to anesthetic and surgical procedures, including blood obstruction and reperfusion, without treatment application.

In all the animals in groups 2, 4, 6, and 8 , two-dimensional ultrasound was used to facilitate the percutaneous application of intrarenal treatments, and the location allowed for the implementation was the 
cortical portion proximal to the caudal pole of the kidney.

\section{Assessment using two-dimensional and color Doppler ultrasound}

Two-dimensional and color Doppler ultrasound examinations were performed in 16 randomly selected animals at 7 days before the surgical procedure to determine patterns of normality of the structural organ and hemodynamics using an ultrasound equipment (GE Logiq-E) with sector transducer 8C (4-11 MHz). In these animals, color Doppler ultrasound was also performed after the placement and removal of the vascular clip, allowing the assessment of renal ischemia and reperfusion.

\section{Histological procedure}

Twenty-four hours after the obstruction of the renal artery and vein, the four animals in group 10 were subjected to hemostatic clip removal and left nephrectomy. In the remaining nine groups, left nephrectomy was performed in four animals to 8 or 31 days after treatment. The removed kidney was sectioned in the sagittal plane, photographed using a digital camera, prepared in histological slides, and stained with hematoxylin and eosin (HE).

The slides with the central sagittal sections were analyzed by optical microscopy to determine the degree of renal injury and necrosis (blind study, without knowledge of the assessed group). The gridded cover slip was placed on the histological section of the tissue, and 20 areas covering the entire tissue extension were defined for histological analysis. The areas of extension of necrosis tubular necrosis, cylinder formation, and tubular dilatation were assessed and assigned scores as follows: 0 (none), $1(\leq 10 \%), 2$ (11\%$25 \%), 3(26 \%-45 \%), 4(46 \%-75 \%)$, and 5 ( $\geq 76 \%)$.
Moreover, the renal tissue slides of 8 or 31 days were analyzed by direct fluorescence microscopy (Floid Cell Imaging Station; Life Technologies) under UV light using specific laser filters to observe cells expressing GFP.

\section{Statistical analysis}

Data tabulation and statistical analysis were performed using Excel $^{\circ}$ spread sheets and the Graphpad Prism software. The values of the variables obtained by histological analysis, namely extension of the tubular necrosis area, cylinder formation, loss of brush border, and tubular dilatation were subjected to the Kolmogorov-Smirnov test to determine the distribution of normality, followed by the Kruskal-Wallis test and Dunn's post-test for multiple comparison. For all statistical data obtained, the rejection of the null hypothesis was set at $5 \%(p \leq 0.05)$.

\section{Results}

In the first 24 hours, the adipose tissue yielded a heterogeneous culture, comprising non adherent rounded cells and lipid micelles in the supernatant. After the $8^{\text {th }}$ day, the cellular population appeared more homogeneous, with the predominance of adherent cells that had a fibroblastoide morphology and were organized in colonies.

Flow cytometry performed on a sample of cultured cells of the fourth passage and GFP revealed that $90.30 \%$ of cells expressed CD90, 86.82\% expressed CD73, 3.02\% expressed CD45, 2.52\% expressed CD11, 23.02\% expressed MHC-I, and $4.50 \%$ expressed MHC-II.

The culture of cells subjected to osteogenic differentiation exhibited calcifications points in the matrix from the tenth day of differentiation induction, and the appearance of the differentiated cells changed from fibroblastoid and elongated to a rounder 
shape. At 30 days, by Von Kossa staining, brownish or blackish mineralization nodules was observed, indicating the presence of extracellular matrix rich in calcium confirming that the cultured ADSC had differentiated into osteoblasts.

In the macroscopic examination after nephrectomy, at 8 and 31 days, the renal parenchyma of kidneys in the sham group exhibited a bright red dish color, similar to that of normal kidneys, with preserved cortical and medullary regions. In addition, their size was similar to that of the contralateral kidneys.

The evaluation of group 10, performed 24 hours after ischemia, revealed ischemic kidneys with a dark red dish color, a congested appearance, a size similar to that of the contralateral kidney, and a weak boundary between the cortical and medullary regions.

At 8 days, a diffuse pale color was observed in the cortical and medullary regions in the groups treated with PBS (groups 1, 2, 5 , and 6), in both periods. In groups 1 and 2, it was not possible to distinguish the cortical region from the medullary region and the kidneys exhibited a slight increase in size in comparison with normal kidneys. In the groups treated with ADSC in 24 hours (groups 3 and 4), the kidneys exhibited focal areas of red dish color in the cortical and medullary regions; the latter were not delimited and there was cortical thickening. In groups 7 and 8 (48 hours), the kidneys showed a normal size and the renal parenchyma had a diffuse bright red dish color, with dark red focal areas in the medullary region.

At 31 days, in groups 1, 2, 5, and 6, the parenchyma showed a diffuse paleness, which was more pronounced in the cortical region. In addition, this region was thinner compared with that of a normal kidney, and the renal regions were delimited (Figure 1A, B, E, and F). In groups 3 and 4 , the kidneys exhibited a normal size and more focal areas of red dish color than those observed in the previous evaluation. The cortical and medullary delimitation was more evident (Figure $1 \mathrm{C}$ and $\mathrm{D}$ ). The samples of group 7 had a diffuse red dish color that was more evident in the medullary region, and there was a weak boundary between the cortical and medullary regions (Figure 1G). In group 8, a bright red dish color was observed in the cortical region, similar to that of normal kidneys, and a less intense color was observed in the medullary region, with retraction of the renal pelvis (Figure $1 \mathrm{H}$ ).

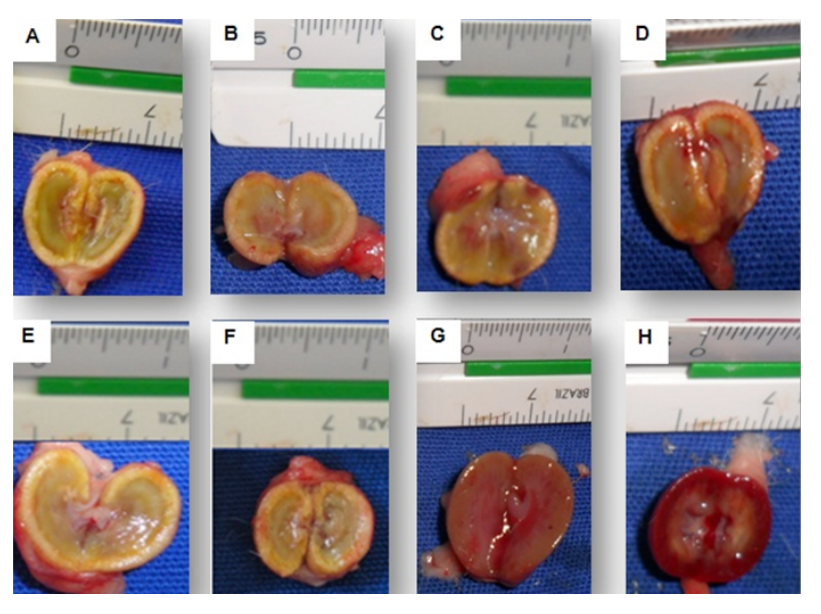

Figure 1 - Photos depicting the macroscopic appearance of the left kidney of Wistar rats subjected to renal artery and vein obstruction at 31 days after treatment. A) Group 1, focal areas with a red dish color. B) Group 2, parenchyma with diffuse pale color, more pronounced in the cortical region. C) Group 3, focal areas with red dish color. D) Group 4, focal areas with red dish color. E) Group 5, parenchyma with diffuse pale color, more pronounced in the cortical region. F) Group 6, parenchyma with diffuse pale color, more pronounced in the cortical region. G) Group 7, diffuse red color, more pronounced in the medullary region, and a weak boundary between the cortical and medullary regions. H) Group 8, bright red dish color in the cortical region and less intense color in the medullary region, with retraction of the renal pelvis. 
Histological examination of the slides of the sham group revealed that the architecture of the renal tubules was preserved, with no areas of necrosis, tubular dilatation, and hyaline cylinders (Figure 2A). The examination of group 10 performed 24 hours after ischemia revealed cell debris inside the tubules, anucleate tubular cells, and non preserved tissue architecture with deformed tubules and glomeruli, corresponding to a necrosis of $5 \%$ of the total area, tubular dilatation of $70 \%$, and formation of hyaline cylinders in $50 \%$ of the tubules (Figure 2B).


Figure 2 - Photomicrography showing renal architecture. A) An animal of the sham group, presence of renal tubules with preserved and uniform architecture and nucleated tubular cells (HE). B) An animal of group 10, presence of tubular dilatation, with accumulation of hyaline cylinders and deformed tubules and glomeruli (HE).

At 8 days, all the slides from the various experimental groups showed renal tubules devoid of the epithelium, filled with cell debris. In addition, reactivity of interstitial cells corresponding to coagulation necrosis was observed, which was more evident in the groups treated with PBS (approximately $90 \%$ in group $1,80 \%$ in group 2, $80 \%$ in group 5, and $90 \%$ in group 6 ) than in the groups treated with ADSC (45\% in group 3, $25 \%$ in group $4,10 \%$ in group 7 , and $40 \%$ in group 8 ).

The number of dilated tubules was higher in the groups treated with PBS $130 \%$ in group $1,45 \%$ in group $2,50 \%$ in group 5 , and $70 \%$ in group 6$)$ than in the groups treated with ADSC (20\% in group $3,40 \%$ in group $4,10 \%$ in group 7 , and $50 \%$ in group 8 ).

The percentages of hyaline cylinders inside tubules in the groups treated with PBS were $20 \%$ in group $1,35 \%$ in group $2,50 \%$ in group 5 , and $10 \%$ in group 6 . In the groups treated with MSC, these values were $20 \%$ in group $3,30 \%$ in group $4,10 \%$ in group 7 , and $10 \%$ in group 8.

At 31 days, the groups treated with PBS exhibited tubular and glomerular necrosis (approximately $90 \%$ in groups 1 and 2 and $100 \%$ in groups 5 and 6). In the groups treated with MSC, the necrotic areas were smaller $150 \%$ in group 3, 40\% in group 4, 30\% in group 7, and $1 \%$ in group 8 ) (Figure 3 ). 

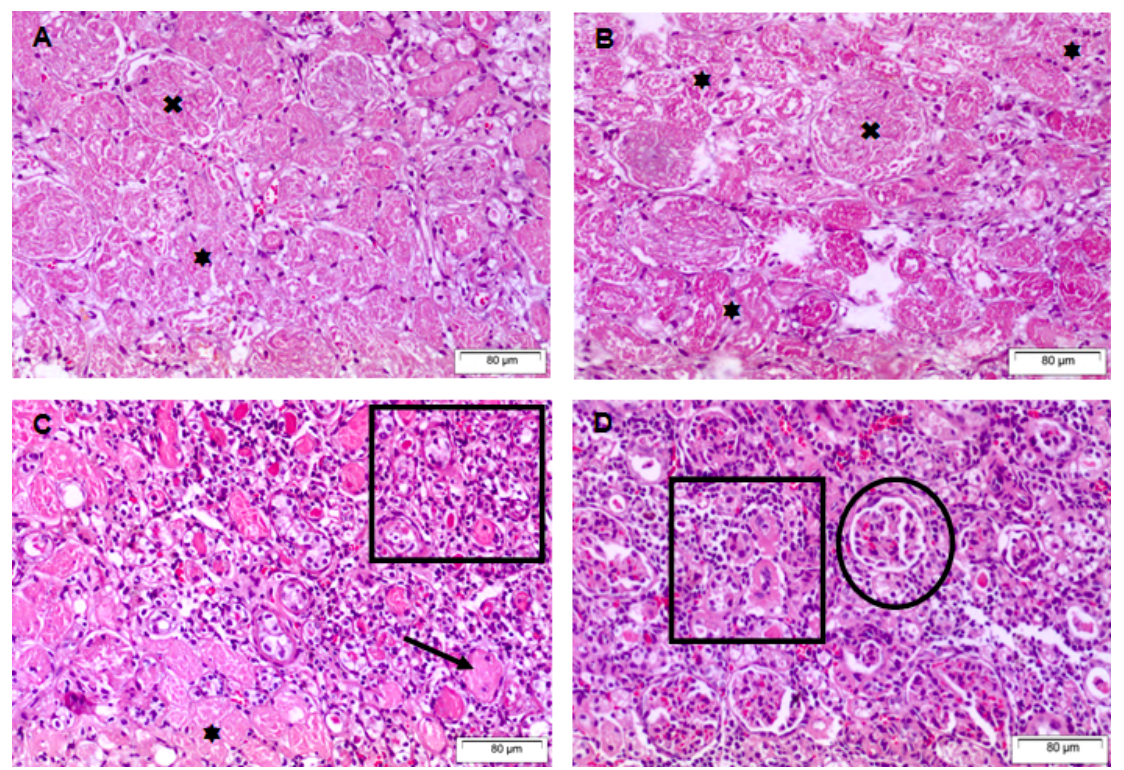

Figure 3 - Renal photomicrography showing histological aspects of the various experimental groups at 31 days. A) Photomicrography showing an animal of group 1. B) Photomicrography showing an animal of group 2. In $A$ and $B$, the images show renal tubules devoid of the epithelium and filled with cell debris, tubular (i) and glomerular (Ó) necrosis, and reactivity of the interstitial cells. C) Photomicrography showing an animal of group 3. Necrotic areas on the left of the field, nucleated tubules (square) with uniform architecture, presence of tubular dilatation (arrow), and formation of hyaline cylinders. D) Photomicrography showing an animal of group 4. Anucleate renal tubules, glomerular organization (circle), and preserved architecture can be observed. E) Photomicrography showing an animal of group 5. F) Photomicrography showing an animal of group 6 . In $E$ and $F$, the images show evident tubular and glomerular necrosis in the entire field, without preservation of the parenchyma architecture. G) Photomicrography showing an animal of group 7. Preservation of the structural architecture of the parenchyma, with nucleated uniform tubules. H) Photomicrography showing an animal of group 8. Vascularization, increased tissue basophilia, and increased number of undifferentiated interstitial cells can be observed. Preserved structural architecture of the parenchyma can also be seen (HE). (escale $=80 \mu \mathrm{m})$

In group 8, there were areas showing a rapid process of regeneration with mitotic figures in tubular and epithelial renal cells, increased number of undifferentiated interstitial cells, and increased number of capillaries. Necrotic tissue replacement by new renal cells was observed.

The percentages of tubular dilatation in the groups transplanted with PBS were $50 \%$ in group 1,90\% in group 2, 50\% in group 5, and $90 \%$ in group 6 . In the groups treated with MSC, these values were $50 \%$ in group 3, 30\% in group 4, 40\% in group 7, and $45 \%$ in group 8. The tubules were dilated; however, the groups transplanted with ADSC exhibited tubules with a smaller circunference than the other groups. In addition, at 31 days, the percentages of hyaline cylinders were $10 \%$ in groups 1,2 , and 5 and $1 \%$ in group 6 . In the groups treated with MSC, these values were $40 \%$ in group 3 , $20 \%$ in group 4 , and $1 \%$ in groups 7 and 8 .

The renal tissue slides of the group treated with ADSC by intrarenal route, 48 hours after injury (group 8) were subjected to immunofluorescence analysis revealed small amounts of cells with green light emission captured by GFP filter in the histological sections performed in 8 days after treatment. In the other groups did not were observed GPF cells, nor even to the 8 to 31 days (Figure 4). 



Figure 4 - Photomicrograph of the renal medullary region under fluorescence microscopy of animals of groups 2 and 8. A) Unspecific fluorescence signal in the green filter in the control group due to processing (formaldehyde and paraffin), forming a homogeneous background. Despite the background, cells with green fluorescence (arrows) were observed in the treated group as a result of the expression of the GFP gene. B) Nuclei stained with Hoechst observed in the blue filter (UV) in both groups. C) Overlap of images of A and B showing the presence of GFP cells in the treated group (arrows). (Magnification $\times 63$ )

Statistical analysis of the variables necrosis, tubular dilatation, and presence of hyaline cylinders revealed that there was a statistically significant difference between the groups treated with ADSC at 8 and 31 days and the groups treated with PBS, regardless of the application route.

With regard to the variable necrosis, there was no difference between the sham group and group 8 at 31 days of observation, which allowed us to infer that the degree of necrosis decreased because there was a statistically significant difference between these groups at 8 days of observation.

The proportion of hyaline cylinders inside the tubules at 31 days in the groups treated with ADSC was higher than that in the untreated groups, which exhibited lower degrees of necrosis. The presence of cylinders is a sign of intracytoplasmic protein accumulation. As tissue degeneration progresses, cylinders are eliminated with the urine.

\section{- Discussion}

The advantages of using ADSC are easy harvesting and the abundance of this tissue in the animal, which enable the collection of a high number of stem cells, as well as the favorable characteristics of culture, expansion, and maintenance through various passages ${ }^{7}$.

The low expression of MHC-I and MHCIl determined by flow cytometry $(23.02 \%$ and $4.50 \%$, respectively) confirms the report of Wan et $a l .{ }^{4}$ and Griffin et $a l .^{8}$ that said the minimal expression of the major histocompatibility complex, MHC-I and MHC-II, explains the low immunogenicity of ADSC. Although the levels of MHC class II are relatively low, the proinflammatory molecules released for ADSC can modulate the immunomodulatory functions in clinical use. Another evidence that rejection did not occur during study was the fact that the animals remained in a stable clinical state during the entire study. 
The two-dimensional and color Doppler ultrasounds provided detailed information on the vascular architecture of the organ and on the presence, direction, and quality of blood flow, thereby enabling renal anatomical and hemodynamic assessment ${ }^{9}$. Moreover, these imaging exams demonstrated the effectiveness of the hemostatic clip in inducing renal ischemia because blood flow was not observed at the moment of occlusion.

Macroscopically, at 8 days, in all the groups, the size of the obstructed kidney was found to be slightly increased compared with that of the contralateral kidney. Tucci-Junior et $a .^{10}$ demonstrated that this increase in size is due to increased mitochondrial respiration activity as a compensatory mechanism of the ischemic organ.

In the groups treated with PBS, at 8 and 31 days of observation, the parenchyma exhibited a diffuse pale color, characteristic of renal infarction progressing to coagulation necrosis. Vessel occlusion resulted in hemorrhagic infarction that initially turned the parenchyma into a yellowish to pale gray color in 2 to 3 days because of erythrocyte lysis and renal hemoglobin excretion; this coloration remained throughout the study ${ }^{11}$.

According to Mota ${ }^{12}$, the most frequent consequence of acute renal disease is acute tubular necrosis. In the present study, at 8 days, in the groups treated with PBS (groups 1, 2,5 , and 6), microscopic changes characteristic of necrosis, such as the presence of cell debris and loss of tubular and glomerular architecture, were observed in practically the entire area of the slides. These findings evolved to intense necrosis, with complete loss of vascularization at 31 days in all the fields examined by microscopy.

After 8 days, the groups treated with ADSC exhibited a lower number of fields with necrotic areas than the groups treated with PBS, and after 31 days, these areas had disappeared. At 31 days, particularly in the animals transplanted via the intrarenal route after 48 hours (group 8), well vascularized regions and areas in the complete process of regeneration were observed, with mitotic figures and glomerular reconstitution, and the architecture was similar to that of the sham group. In recent studies, Chen et al. ${ }^{13}$ too observed that treatment with ADSC after ischemia-reperfusion- injury induced helped to reestablish of the architectural integrity of the renal parenchyma and angiogenesis.

The results of the groups that received ADSC via the intrarenal route were better than those of the groups that received ADSC via the intravenous route through inoculation in the tail vein. Transplantation at the site of injury allowed a higher concentration of undifferentiated cells to interact with the host tissue, with cell signaling occurring via growth factors and exerting the paracrine and telecrine effects on the remaining cells of the body ${ }^{3,4,14}$. In addition, the remaining cells of the organ were stimulated to differentiate, proliferate and mobilize ${ }^{15}$, there by contributing to the cellular reconstitution of the tubular epithelium ${ }^{16}$ to preserve the integrity of the renal parenchyma. However, compared with the control, the group that received the venous infusion also exhibited consistent improvement. This improvement combined with the ease of application of this type of therapy makes this route highly indicated for clinical treatments with stem cells.

Groups 7 and 8 showed better responses than groups 3 and 4 with regard to morphological and functional kidney restoration. This allowed us to infer that after 48 hours, the transplanted ADSC interacted better with the host's niche when the inflammatory response of the injured tissue had already initiated. Moreover, in this initial phase, which is present in all processes of tissue repair, chemical mediators are 
released, e.g., interferon gamma (INF- $\gamma$ ), which intensifies the interaction of ADSC with the niche, thereby increasing paracrine signaling and cell recruitment ${ }^{17}$.

By immunofluorescence analysis was observed few amount cells with GFP expression in the histological analysis of group 8 in the moment of evaluation. However, the structural and functional improvement observed this group leads us to infer that the largest action of GFP cells occurred by telecrine mechanism. According to Lindoso et al. ${ }^{18}$, this observation is correct because cellular reconstitution is not expected via the differentiation of ADSC but rather via the functional restoration of local cells mediated by the systemic immunomodulatory effects exerted by the transplanted undifferentiated cells. Possibly, if we had realized immunofluorescence of animals lung tissue receiving intravenous MSC infusion could visualize GFP cells in evaluation histological sections of 8 day because according to Zhang et al. ${ }^{19}$, almost all ADSC administered intravenously can migrate and accumulate in the lung parenchyma within 11 days after the infusion.

\section{Conclusions}

Allogenic transplantation of adipose tissue-derived MSC positively contributed to the replacement of the necrotic tissue by renal tubular cells, vascularization of the renal parenchyma, and functional restoration of the organ. Transplantation via the intrarenal route was more effective than transplantation via the intravenous route in organ restoration, and transplantation at 48 hours after injury showed better results than transplantation at 24 hours. The group that received the venous infusion also exhibited consistent improvement in the renal function and this improvement combined with the ease of application of this type of therapy makes this route highly indicated for clinical treatments with stem cells.

\section{References}

1- Humphreys BD, Bonventre JV. Mesenchymal stem cells in acute kidney injury. Ann Rev Med. 2008;59:311-25. doi:10.1146/ annurev.med.59.061506.154239.

2- Thadhani R, Pascual M, Bonventre JV. Acute renal failure. New Eng J Med. 1996;334:144860. doi: 10.1056/NEJM199605303342207.

3- Bi B, Schmitt $R$, Israilova $M$, Nishio $H$, Cantley LG. Stromal cells protect against acute tubular injury via an endocrine effect. J Am Soc Nephrol. 2007;18:2486-96. doi: 10.1681/ASN.2007020140.

4- Wan CD, Wang HB, Liu T. Immunomodulatory effects of mesenchymal stem cells derived from adipose tissues in a rat orthotopic liver transplantation model. Hepatobiliary Pancreat Dis Int. 2008;7:29-33. PMDI: 18234635.

5- Del Carlo RJ, Monteiro BS, Argolo-Neto NM. Avanços no estudo de células- tronco no Brasil e suas implicações. Ceres. 2009;56:446-50.

6- Monteiro BS, Favarato $L$, Carvalho $P$, Okano B, Menegatti MA, Oliveira A, Santos B, Del Carlo, R. Clinical and radiographic characterization of xenotransplantation of rat bone marrow-derived mesenchymal stem cells for repair of radial defects of rabbit. Turk J Vet Anim Sci. 2015;39:511-9. doi: 10.3906/vet-1405-72.

7- Zuk PA, Zhu M, Ashjian P, De Ugarte DA, Huang JI, Mizuno $\mathrm{H}$, Alfonso ZC, Fraser JK, Benhaim $\mathrm{P}$, Hedrick $\mathrm{MH}$. Human adipose tissue is a source of multipotent stem cells. Mol Bio Cell. 2002;13:4279-95. doi: 10.1091/mbc.e02-02-0105.

8- Griffin MD, RitterT, Mahon BP.Immunological aspects of allogeneic mesenchymal stem cell therapies. Hum Gene Ther. 2010;21:164155. doi: 10.1089/hum.2010.156.

9- Engelhorn CA, Engelhorn AL, Pulling R. Vascular color Doppler ultrasound in the evaluation of renovascular hypertension. Accuracy of the direct technique of renal artery assessment. Arq Bras Cardiol. 2004;82:473-6. doi: 10.1590/S0066782X200400050001.

10-Tucci-Junior S, Molina CAF, Cologna AJ, Suaid HJ, Tirapelli LF, Celini FM, Martins ACP. Compensatory renal growth and mitochondrial function: the influence of 
warm ischemia and reperfusion. Acta Cir Bras. 2008;23:31-5. doi: 10.1590/S010286502008000700006.

11-Confer AW, Panciera RJ. Urinary System. In: Carlton WW, McGavin MD. Special veterinary pathology. Porto Alegre: Ed. Arte Médica; 1998. p.228-65.

12-Mota PC. Indicações actuais para biópsia renal. Acta Med Port. 2005;18:147-51.

13-Chen Y, Sun CK, Lin YC, Chang LT, Chen YL, Tsai TH, Chung SY, Chua S, Kao YH, Yen $\mathrm{CH}$, Shao PL, Chang KC, Leu S, Yip HK. Adiposederived mesenchymal stem cell protects kidneys against ischemia reperfusion injury through suppressing oxidative stress and inflammatory reaction. J Transl Med. 2011;9:1-17. doi: 10.1186/1479-5876-9-51.

14-Feng Z, Ting J, Alfonso Z, Strem BM, Fraser JK, Rutenberg J, Kuo HC, Pinkernell K. Fresh and cryopreserved, uncultured adipose tissue-derived stem and regenerative cells ameliorate ischemia-reperfusion-induced acute kidney injury. Nephrol Dial Transplant. 2010;25:3874-84. doi: 10.1093/ndt/gfq603.

15-Kopp HG, Avecilla ST, Hooper AT, Rafii S. The bone marrow vascular niche: home of HSC differentiation and mobilization. Physiology. 2005;20:349-56. doi: 10.1152/ physiol.00025.2005.

16-Kim J, Lim JI, Na YK, Park KM. Intra-renal slow cell-cycle cells contribute to the restoration of kidney tubules injured by ischemia/ reperfusion. Anat Cell Biol. 2011;44:186-93. doi: 10.5115/acb.2011.44.3.186.

17-Fuchs, E, Tumbar T, Guasch G. Socializing with the neighbors: stem cells and their niche. Cell. 2004;116:769-78. doi: 10.1016/ S0092-8674(04)00255-7.

18-Lindoso RS, Araújo DS, Novaes JA, Mariante RM, Verdoorn KS, Fragel-Madeira L, CarusoNeves C, Linden R, Vieyra A, EinickerLamas $M$. Paracrine interaction between bone marrow derived stem cells and renal epithelial cells. Cell Physiol Biochem. 2011;28:267-78. doi: 10.1159/000331739.

19-Zhang TY, Huang B, Yuan ZY, HU YL, Tabata Y, Gao JQ. Gene recombinant bone marrow mesenchymal stem cells as a tumor-targeted suicide gene delivery vehicle in pulmonary metastasis therapy using non-viral transfection. Nanomedicine. 2014;10:25767. doi: 10,1016/j.nano.2013.06.003.

\section{Correspondence:}

Betânia Souza Monteiro

Laboratório de Célula-Tronco e Terapia Celular,

Universidade Vila Velha

Avenida Comissário José Dantas de Melo, 21

29102-920 Velha-ES Brasil

Tel.: (55 27)98155-5700

betania.monteiro@uvv.br

betania.monteiro@gmail.com

Received: July 22, 2018

Review: Sept 29, 2018

Accepted: Oct 25, 2018

\section{Conflict of interest: none}

Financial sources: CNPq (Process 552488/2011-

9 and 483518/2011-5), and FAPES
${ }^{1}$ Research performed at Stem Cell and Cellular Therapy Laboratory, and Veterinay Hospital, Universidade Vila Velha (UVV), Brazil. 